\title{
Parametric Construction of Improved Nyquist Filters Based on Inner and Outer Functions
}

\author{
Stylianos D. Assimonis*, Michail Matthaiou ${ }^{\dagger}$, George K. Karagiannidis*, and Josef A. Nossek ${ }^{\dagger}$ \\ *Department of Electrical and Computer Engineering, Telecommunications Division, \\ Aristotle University of Thessaloniki, 540 06, Thessaloniki, Greece \\ email:\{asimonis,geokarag\}@ auth.gr \\ †Institute for Circuit Theory and Signal Processing, Technische Universität München (TUM), \\ Arcistrasse 21, 80 333, Munich, Germany \\ email:\{matthaiou,nossek\}@nws.ei.tum.de
}

\begin{abstract}
In this paper, we explore the concept of inner and outer functions to come up with two novel parametric families of Nyquist pulses. Aside from requiring only two design parameters, the proposed pulses yield an enhanced performance compared to the sophisticated flipped-inverse hyperbolic secant (asech) pulse, that was recently presented in the literature. While the construction of parametric families originates from the work of Beaulieu and Damen, the usage of inner and outer functions guarantees a higher flexibility in the choice of the composite family members. The proposed pulses may have a slower decay rate than the well-known raised-cosine (RC) pulse, but exhibit a more pronounced decrease in the amplitudes of the two largest sidelobes and this accounts for their improved robustness to error probabilities. In the following, it is clearly demonstrated that a lower bit error rate (BER), compared to the existing pulses, can be achieved for different values of the roll-off factor and timing jitter. Moreover, a smaller maximum distortion along with a more open eye diagram are attained as well.
\end{abstract}

\section{INTRODUCTION}

The rapid growth of digital communications over the last decades imposes better bandwidth reuse and higher error-free data rates. The most popular Nyquist pulse for distortionless transmission without the presence of intersymbol interference (ISI) is the RC pulse which is basically a low-pass filter with odd symmetry around a cutoff frequency and has a cosine shaped roll-off portion [1]. Apart from the ISI-free prerequisite, pulse-shaping filters have to exhibit low sensitivity to timing errors [2]. Nevertheless, in practical receivers the presence of timing jitter causes the actual sampling points to deviate from the optimal positions thereby leading to symbol timing errors. This, in turn, implies that the pulse tails must decay as quickly as possible outside the pulse interval so that the undesired effects of jitter are eliminated. What's more, taking into account that the great majority of current practical systems are band-limited along with the steadily increasing spectrum congestion, it becomes apparent that the system designer has to convey the highest possible errorfree data rate within a fixed limited bandwidth. Normally, a tradeoff exists between increased data rate and time-domain tail suppression [2].

In order to meet all the above mentioned constraints a plethora of alternatives have been reported in the corresponding literature. In [3], the so-called Beaulieu or better than raised cosine pulse (BTRC) pulse, which outperforms the $\mathrm{RC}$ pulse in terms of larger eye opening and smaller average symbol error rate (SER), was proposed. This performance enhancement was observed even though the tails of the BTRC pulse asymptotically decay as $1 / t^{2}$ while those of RC as $1 / t^{3}$. This interesting phenomenon can be attributed to the fact that the two main sidelobes' amplitudes of BTRC are smaller than the largest sidelobes' amplitudes of RC. Please note that the BTRC pulse remains the best pulse known with an explicit time-domain formula. Other superior pulses that do not have an explicit time-domain expression, have also been devised; in particular, the authors in [4] developed the so-called flippedhyperbolic secant (fsech) and flipped-inverse hyperbolic secant (asech) ${ }^{1}$ pulses; the latter performs even better than the BTRC and yields a smaller maximum distortion and also remains robust to root and truncation operations. Please note that throughout the paper it will be our reference pulse since, to the best of our knowledge, yields the best performance compared to any other pulse available in the literature with two design parameters.

From the previous discussion, we can infer that a pulse with the minimum number of design parameters and smallest BER for any arbitrary value of the roll-off factor and sampling time error, represents the key goal in the design of practical receivers. In this light, two novel parametric ISI-free families are presented herein with only two design parameters that exhibit better error performance and a more open eye-diagram than the sophisticated asech pulse. It is also shown that the latter is a member of the first proposed family which in practice means that the proposed family represents a generalization of the work presented in [4].

The main motivation of the following analysis has been the pioneering work carried out by Beaulieu and Damen in [5]. In their paper, the authors proposed a parametric family of Nyquist ISI-free pulses based on a spline frequency domain pattern with the main advantage lying in the high flexibility in the pulse design. The members of this family have an even and real frequency spectrum which implies that the time pulse

\footnotetext{
${ }^{1}$ In the original paper [4], this pulse was referred to as farcsech. We use the name asech instead for the continuity of notation throughout the paper.
} 
is real valued and even, respectively. However, the flexibility in finding the optimum pulse for each specific application is a result of a third free parameter which increases the degrees of freedom, thereby making most of the known ISI-free pulses represent special cases of this generalized family.

The remainder of the paper is organized as follows: In Section II, we introduce the two parametric families of ISI-free pulses in the frequency domain. In Section III, the performance of both families is evaluated from different practical perspectives of interest. Finally, Section IV concludes the paper and summarizes the key findings.

\section{PARAmetric Families of ISI-Free Pulses Using INNER AND OUTER FUNCTIONS}

The proposed families have been built upon the notions of inner and outer functions which find numerous applications spanning wavelet theory to image compression and modeling of microwave circuits [6]. In the present case, the main concept for the filter construction is the following:

1) Choose an outer function $g(f)$ so that it only needs to be continuous and differentiable in the frequency interval $B(1-\alpha) \leq|f| \leq B(1+\alpha)$ and, more importantly, its frequency response retains a concave shape in the interval $B(1-\alpha)<|f| \leq B$ and convex in $B<|f| \leq B(1+\alpha)$. We recall that both RC and fsech pulses have a convex shape in $B(1-\alpha)<|f| \leq B$ and concave in $B<|f| \leq B(1+\alpha)$. Two candidate trigonometric functions that fulfill the aforementioned conditions are the well-known inverse cosine and inverse hyperbolic secant functions which can be denoted as $g(f)=\operatorname{acos}(f)$ and $g(f)=\operatorname{asech}(f)$, respectively.

2) Once the outer function $g(f)$ has been chosen, the different choices of the inner functions $h(f)$ represent essentially tradeoffs between the decay rate and the sidelobes' amplitudes and for this reason yield different BERs and eye diagrams. Then, the composite filter response can be written as $\mathcal{G}(f)=g(h(f))$.

For the first proposed family of filters, three different alternatives (members) are introduced, according to $h(f)=$ $\operatorname{acos}(f), h(f)=\operatorname{asech}(f)$ and $h(f)=\log (f)$. Then, the composite filter's frequency response reads as

$$
\begin{aligned}
& S_{1}(f)= \\
& \begin{cases}T, & |f| \leq B(1-\alpha) \\
T\left\{1-\frac{1}{2 \gamma} \mathcal{G}\left(\frac{\gamma_{0}}{2 \alpha B}(-f+B(1+\alpha))\right)\right\}, & B(1-\alpha)<|f| \leq B \\
T\left\{\frac{1}{2 \gamma} \mathcal{G}\left(\frac{\gamma_{0}}{2 \alpha B}(f-B(1-\alpha))\right)\right\}, & \\
0, & B<|f|<B(1+\alpha) \\
& B(1+\alpha) \leq|f|\end{cases}
\end{aligned}
$$

where $\alpha$ denotes the roll-off factor $(0 \leq \alpha \leq 1)$, determining the bandwidth occupied by the pulse and the time-domain tail suppression, while $B=1 /(2 T)$ is the Nyquist frequency and $T$ the repetition rate (transmission symbol period). The parameters $\gamma$ and $\gamma_{0}$ are defined directly through the inverse $\mathcal{G}$ function according to

$$
\gamma_{0}=\mathcal{G}^{-1}(0) \quad \text { and } \quad \gamma=\mathcal{G}\left(\frac{\gamma_{0}}{2}\right) .
$$

This choice ensures the continuity of $S(f)$ within the considered interval $[0,(1+\alpha)]$ and further at the key transition points

$$
f=B(1-\alpha), \quad f=B \text { and } f=B(1+\alpha)
$$

the obtained values are respectively

$$
S(B(1-\alpha))=1, \quad S(B)=1 / 2 \text { and } S(B(1+\alpha))=0 .
$$

It is noted that the asech pulse is by definition a special member of this family, obtained as $\mathcal{G}(f)=\operatorname{asech}(f)$. In a similar manner, we obtain the acos pulse $\mathcal{G}(f)=\operatorname{acos}(f)$ which represents essentially the inverse of the RC Nyquist pulse. Henceforth, each composite family member will be denoted via the inner and outer functions, separated by brackets, i.e. $\operatorname{acos}[\mathrm{acos}]$ and so on. The frequency responses of all the possible combinations are depicted in Fig. 1(a) and 1(b) for a roll-off factor $\alpha=0.35$.

The second proposed family has only two members and the exponential function as child function. The main reason behind developing this separate family was the fact that when $h(f)=\exp (f)$ we readily obtain $\gamma_{0}=0$; then, (1) reduces into a rectangular pulse in the interval $[-B, B]$. In order to circumvent this undesired implication, we also propose a second class of Nyquist filters, that is

$$
\begin{aligned}
& S_{2}(f)=
\end{aligned}
$$

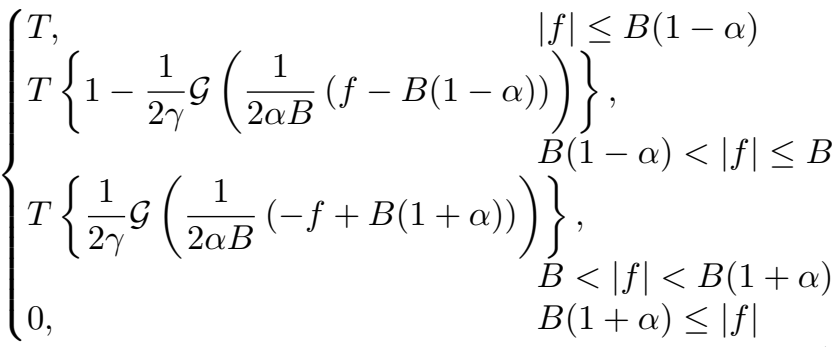

where $\gamma=\mathcal{G}\left(\frac{1}{2}\right)$. The frequency response of the second family is illustrated in Fig. 2. As was previously mentioned, both $S_{1}(f)$ and $S_{2}(f)$ have a concave shape in the interval $B(1-\alpha)<|f| \leq B$ and convex in the interval $B<|f| \leq B(1+\alpha)$, in order to allow for an amount of energy to be transferred in the high spectral region. Under these circumstances and after observing that the frequency responses are continuous functions while their first derivatives are discontinuous, it is found ${ }^{2}$ that the impulse responses' tails asymptotically decay as $1 / t^{2}$.

\footnotetext{
${ }^{2} \mathrm{~A}$ detailed proof can be found in an extended journal version of this paper [7].
} 


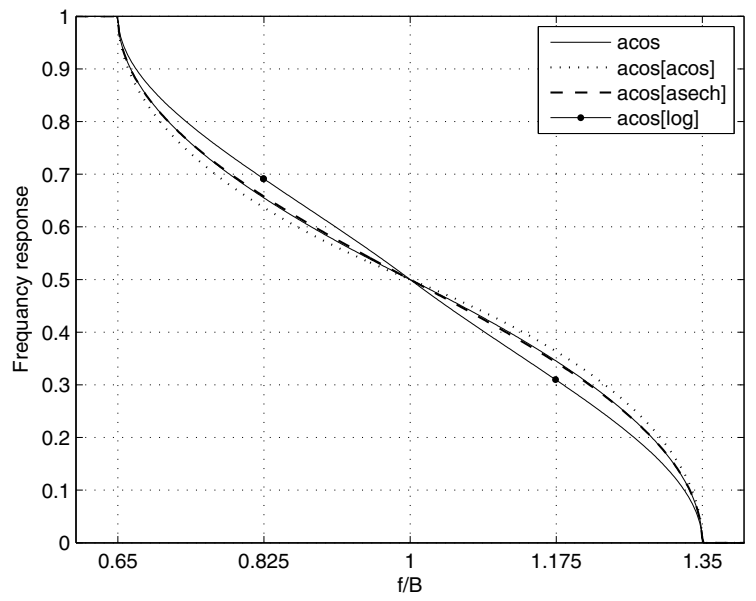

(a) Acos filters.

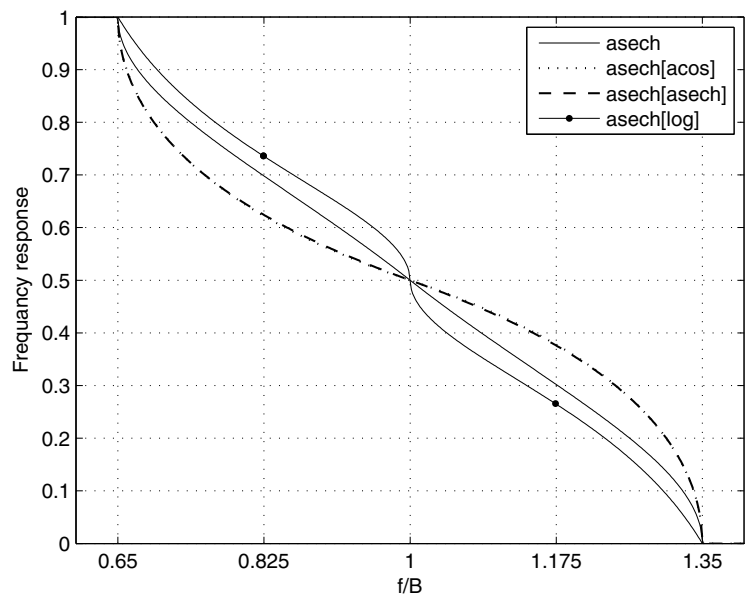

(b) Asech filters.

Fig. 1. Frequency-domain responses of the first parametric family (acos and asech filters) with roll-off factor $\alpha=0.35$.

\section{PERFormance EVAluation}

In this section, our primary goal is to evaluate the performance of the proposed families from different practical perspectives of interest. We begin with the comparison of the eye diagrams which are a means of visually assessing the vulnerability of transmission systems to the problem of ISI [2]. For the sake of brevity, we consider only the eye diagrams of the reference asech pulse, originally given in [4], and that of the $\operatorname{acos}[\log ]$ pulse which yields the best performance among all the proposed filters. The corresponding eye patterns, which were generated by superimposing $2^{9}$ distinct binary pulse sequences and observing two consecutive symbol periods, can be seen in Fig. 3. A slightly reduced eye opening is provided by the asech pulse which implies a higher level of ISI and, consequently, an increased sensitivity to synchronization errors [2].

In order to get a deeper insight, the eye width of all the proposed pulses has been estimated at a timing offset of $t / T=$

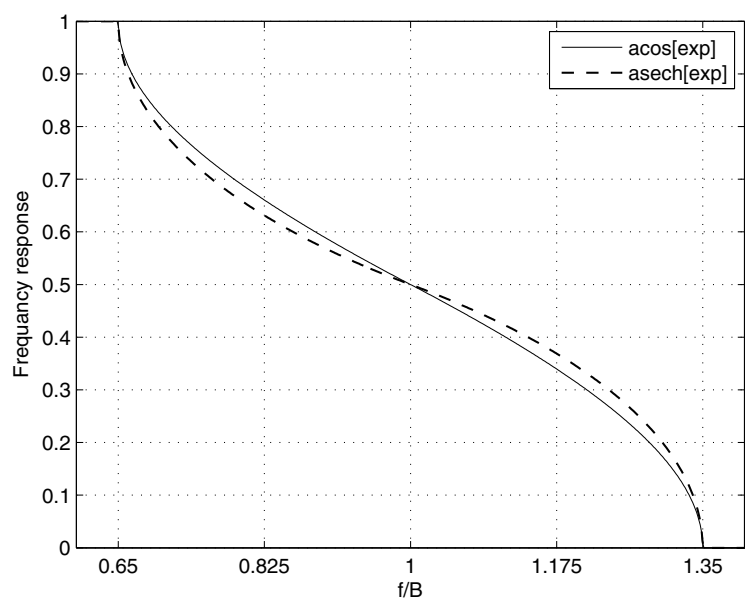

Fig. 2. Frequency-domain responses of the second parametric family with roll-off factor $\alpha=0.35$.
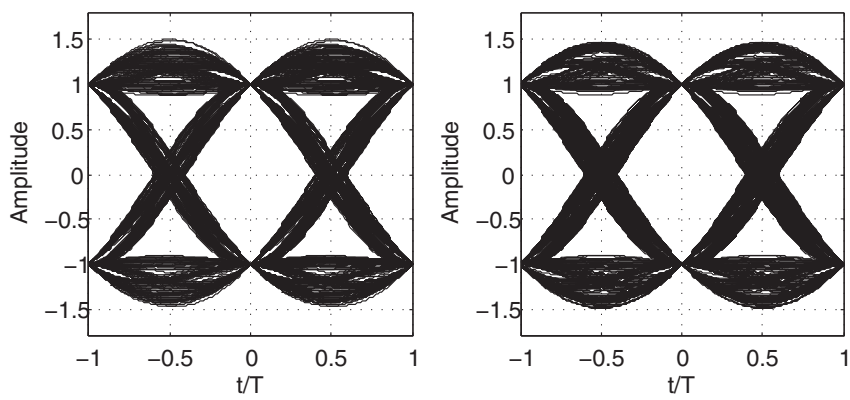

Fig. 3. Eye diagrams of the asech (left) and acos[log] (right) pulses with roll-off factor $\alpha=0.35$.

0.5 , as in [5]. The obtained results are tabulated in Table I (columns 2 and 5). It is interesting to note that the largest eye opening is returned by the acos[log] pulse followed by the asech[exp] pulse. The reference asech pulse may perform better than the majority of the proposed pulses in terms of eye opening but, as it will be shown in the following, it falls short of offering a satisfactorily low BER.

Consistent conclusions can be drawn after estimating the maximum distortion of each separate pulse which is a more quantitative measure of performance (see columns 3 and 6 in Table I). We recall that the maximum distortion is the magnitude of the largest possible ISI sample at any given time instant. The point of maximum distortion occurs always at $t / T= \pm 0.5$ and the best performance is offered by the acos pulse with the associated value being 1.4387 . For the second family, asech[exp] yields a value of 1.5093 and, hence, outperforms acos[exp]. The reference asech pulse yields a maximum distortion of 1.4880, which makes it be outperformed by $\operatorname{acos}[\mathrm{asech}], \operatorname{acos}[\log ]$ and asech[acos] while the rest pulses yield a relatively higher value of distortion.

The last step of the evaluation process comprises the computation of the average BERs in the presence of time sampling errors. According to [8], the BER probability is 
TABLE I

Eye Width And Maximum Distortion Of Eye Diagrams Of All Filters

\begin{tabular}{|c|c|c|c|c|c|}
\hline pulse & eye width & max distortion & pulse & eye width & max distortion \\
\hline $\operatorname{acos}$ & 0.5365 & 1.4387 & asech & 0.5983 & 1.4880 \\
\hline $\operatorname{acos}[\mathrm{acos}]$ & 0.5211 & 1.4923 & $\operatorname{asech}[\operatorname{acos}]$ & 0.4922 & 1.4809 \\
\hline $\operatorname{acos}[\operatorname{asech}]$ & 0.5663 & 1.4770 & asech[asech] & 0.5113 & 1.5132 \\
\hline $\operatorname{acos}[\log ]$ & 0.6105 & 1.4663 & asech $[\log ]$ & 0.5891 & 1.5125 \\
\hline $\operatorname{acos}[\exp ]$ & 0.4990 & 1.5256 & $\operatorname{asech}[\exp ]$ & 0.6030 & 1.5093 \\
\hline
\end{tabular}

TABLE II

Bit Error Probability of Both Families of Filters For $N=2^{9}$ INTERFERING Symbols AND SNR $=15$ DB

\begin{tabular}{c||c||cccc}
$\alpha$ & pulse & $t / T= \pm 0.05$ & $t / T= \pm 0.1$ & $t / T= \pm 0.2$ & $t / T= \pm 0.3$ \\
\hline \hline \multirow{4}{*}{0.35} & acos & $3.3527 \mathrm{e}-8$ & $3.9249 \mathrm{e}-7$ & $6.5764 \mathrm{e}-5$ & $4.1127 \mathrm{e}-3$ \\
& acos[acos] & $3.2753 \mathrm{e}-8$ & $3.7964 \mathrm{e}-7$ & $6.4348 \mathrm{e}-5$ & $4.0152 \mathrm{e}-3$ \\
& acos[asech] & $3.3558 \mathrm{e}-8$ & $3.9255 \mathrm{e}-7$ & $6.5582 \mathrm{e}-5$ & $4.1067 \mathrm{e}-3$ \\
& acos[log] & $3.5470 \mathrm{e}-8$ & $4.3365 \mathrm{e}-7$ & $7.3486 \mathrm{e}-5$ & $4.5509 \mathrm{e}-3$ \\
\hline \multirow{3}{*}{0.5} & acos & $2.0431 \mathrm{e}-8$ & $1.3300 \mathrm{e}-7$ & $1.4717 \mathrm{e}-5$ & $1.2578 \mathrm{e}-3$ \\
& acos[acos] & $2.0054 \mathrm{e}-8$ & $1.3014 \mathrm{e}-7$ & $1.5328 \mathrm{e}-5$ & $1.3642 \mathrm{e}-3$ \\
& acos[asech] & $2.0438 \mathrm{e}-8$ & $1.3273 \mathrm{e}-7$ & $1.4563 \mathrm{e}-5$ & $1.2421 \mathrm{e}-3$ \\
& acos[log] & $2.1559 \mathrm{e}-8$ & $1.4514 \mathrm{e}-7$ & $1.4987 \mathrm{e}-5$ & $1.2082 \mathrm{e}-3$ \\
\hline \multirow{4}{*}{0.35} & asech & $3.5970 \mathrm{e}-8$ & $4.4580 \mathrm{e}-7$ & $7.6203 \mathrm{e}-5$ & $4.6950 \mathrm{e}-3$ \\
& asech[acos] & $3.2264 \mathrm{e}-8$ & $3.7363 \mathrm{e}-7$ & $6.4494 \mathrm{e}-5$ & $4.0024 \mathrm{e}-3$ \\
& asech[asech] & $3.2255 \mathrm{e}-8$ & $3.7275 \mathrm{e}-7$ & $6.4110 \mathrm{e}-5$ & $3.9850 \mathrm{e}-3$ \\
& asech[log] & $4.0693 \mathrm{e}-8$ & $5.8264 \mathrm{e}-7$ & $1.1261 \mathrm{e}-4$ & $6.4252 \mathrm{e}-3$ \\
\hline \multirow{3}{*}{0.5} & asech & $2.1875 \mathrm{e}-8$ & $1.4916 \mathrm{e}-7$ & $1.5345 \mathrm{e}-5$ & $1.2253 \mathrm{e}-3$ \\
& asech[acos] & $1.9865 \mathrm{e}-8$ & $1.2958 \mathrm{e}-7$ & $1.6248 \mathrm{e}-5$ & $1.4975 \mathrm{e}-3$ \\
& asech[asech] & $1.9845 \mathrm{e}-8$ & $1.2902 \mathrm{e}-7$ & $1.6057 \mathrm{e}-5$ & $1.4781 \mathrm{e}-3$ \\
& asech[log] & $2.5129 \mathrm{e}-8$ & $2.0062 \mathrm{e}-7$ & $2.2804 \mathrm{e}-5$ & $1.7104 \mathrm{e}-3$ \\
\hline \hline \multirow{2}{*}{0.35} & acos[exp] & $3.3806 \mathrm{e}-8$ & $3.9786 \mathrm{e}-7$ & $6.6617 \mathrm{e}-5$ & $4.1638 \mathrm{e}-3$ \\
& asech[exp] & $3.2591 \mathrm{e}-8$ & $3.7775 \mathrm{e}-7$ & $6.4445 \mathrm{e}-5$ & $4.0128 \mathrm{e}-3$ \\
\hline \multirow{2}{*}{0.5} & acos[exp] & $2.0583 \mathrm{e}-8$ & $1.3446 \mathrm{e}-7$ & $1.4657 \mathrm{e}-5$ & $1.2385 \mathrm{e}-3$ \\
& asech[exp] & $1.9992 \mathrm{e}-8$ & $1.3005 \mathrm{e}-7$ & $1.5658 \mathrm{e}-5$ & $1.4094 \mathrm{e}-3$ \\
\hline
\end{tabular}

the ultimate measure of performance that includes the effects of noise, synchronization and distortion. The error rates have been estimated according to [9] for binary antipodal signaling. A system Signal-to-Noise ratio (SNR) of $15 \mathrm{~dB}$ has been assumed while $2^{9}$ interfering symbols are generated for all pulses. The obtained results are tabulated in Table II. Generally speaking, timing jitter raises the values of average BER since ISI is a result of the receiver eye being sampled off center [3]. It can be easily observed that for most values of $\alpha$ and timing jitter, all the proposed pulses yield a smaller BER than asech. This trend is more pronounced for small and moderate values of $\alpha$ and $t / T$, as commonly encountered in practice.

More importantly, by inspection of Tables I and II, it can be conjectured that the best pulse is asos[log] since it yields not only the largest eye opening but also outperforms asech in terms of maximum distortion and average BER, for all the eight considered cases. On the other hand, asech[log] is systematically outperformed by asech and this phenomenon can be attributed to the higher time-domain sidelobes of the former (see Fig. 1). The rest pulses offer a superior performance for either seven (acos, $a \cos [\mathrm{acos}], \mathrm{acos}[\mathrm{asech}]$ and $\operatorname{acos}[\exp ]$ ) or six cases (asech[acos], asech[asech] and asech[exp]), thereby indicating the improved robustness of the proposed families of filters.

\section{CONCLUSION}

In the present contribution, two novel parametric families of ISI-free pulses were devised that systematically outperform the asech pulse, as developed by Assalini and Tonello. Apart from their improved performance in terms of BER and maximum distortion for the great majority of cases, an important advantage of these families is their high flexibility when it comes down to the construction of a specific pulseshaping filter. After introducing the concept of inner and outer functions, we firstly employed acos and asech as the outer functions in order to guarantee that the frequency responses are concave in the interval $B(1-\alpha)<|f| \leq B$ and convex in $B<|f| \leq B(1+\alpha)$. Then, a plethora of alternatives can be 
potentially obtained by simply changing the inner functions. All the considered pulses have an asymptotic time-domain decay rate of $1 / t^{2}$ which is lower than that of $\mathrm{RC}$ and fsech pulses; despite this fact, an improved performance was still observed since the amplitudes of the two higher sidelobes are lower and this results in a smaller BER. We finally underline the reduced complexity of the proposed families which, contrary to the majority of ISI-free pulses available in the literature, require only two design parameters (degrees of freedom). For this reason, they have a theoretical and practical interest when it comes down to the design of real-life robust receivers.

\section{REFERENCES}

[1] H. Nyquist, "Certain topics in telegraph transmission theory," AIEE Transactions, vol. 47, pp. 617-644, 1928.
[2] J. G. Proakis, Digital Communications, 4th edition, New York: McGrawHill, 2000.

[3] N. C. Beaulieu, C. C. Tan, and M. O. Damen, "A "better than" Nyquist pulse," IEEE Commun. Lett., vol. 5, no. 9, pp. 367-368, Sept. 2001.

[4] A. Assalini and A. M. Tonello, "Improved Nyquist pulses," IEEE Commun. Lett., vol. 8, no. 2, pp. 87-89, Febr. 2004.

[5] N. C. Beaulieu and M. O. Damen, "Parametric construction of NyquistI pulses," IEEE Trans. Commun., vol. 52, no. 12, pp. 2134-2142, Dec. 2004.

[6] S. Theodoridis and K. Koutroumbas, Pattern Recongition: 2nd Edition, Acamedic Press, Inc., 2003.

[7] S. D. Assimonis, M. Matthaiou, G. K. Karagiannidis, and J. A. Nossek "Improved parametric families of ISI-free Nyquist pulses using inner and outer functions," in preparation.

[8] C. C. Tan and N. C. Beaulieu, "Transmission properties of conjugate-root pulses," IEEE Trans. Commun., vol. 52, no. 4, pp. 553-558, Apr. 2004.

[9] N. C. Beaulieu, "The evaluation of error probabilities for intersymbol and cochannel interference," IEEE Trans. Commun., vol. 31, pp. 1740-1749, Dec. 1991. 